\title{
SỬ DỤNG MẠNG NEURON HOPFIELD TĂNG CƯỜNG Độ CHÍNH XÁC NHận DẠNG ĐỐI TƯợNG CÓ KÍCH THƯớC NHỎ HƠN 1 PIXEL TRÊN ẢNH PHỔ
}

\author{
ĐẶNG THANH TÙNG ${ }^{(1,2)}$, ĐÕ̃ NHU’ HIỆP(2), PHẠM ĐİNH TỈNH ${ }^{(2)}$, \\ ĐỖ MẠNH HÀ̀ ${ }^{(3)}$, NGUYẼ̃N THẾ LUÂN ${ }^{(4)}$, LƯONG TH!̣ KIM DUNG ${ }^{(5)}$, LÝ THU HẦNG(6) \\ ${ }^{(1)}$ State Key Laboratory of Information Engineering in Surveying, Mapping and Remote Sensing (LIES- \\ MARS), Wuhan University, 129 Luoyu Road, Wuhan 430079, China \\ ${ }^{(2)}$ Đại học Tài nguyên và Môi trường Hà Nộ \\ ${ }^{(3)}$ Cuc Đo đạc Bản đồ và Thông tin Địa lý Việt Nam \\ (4)Viện Địa chất và Địa vật lý biển-Viện Hàn lâm Khoa học và Công nghệ Việt Nam \\ ${ }^{(5)}$ Truoòng Cao đẳng Vĩnh Phúc \\ ${ }^{(6)}$ Trung tâm Dũ liệu và Thông tin Đất đai-Tổng cuc Quản lý Đất đai
}

\section{Tóm tắt:}

Trong lĩnh vực viễn thám, phân loại mềm được coi là có độ chính xác cao hơn phân loại cứng. Đối với bài toán siêu phân giải, mạng neuron Hopfield đã được sử dụng để thực hiện bài toán tối uu hoá trong xác định vị trí của các lớp phủ trong mỗi pixel. Nghiên cứu này đánh giá sư hiệu quả của thuật toán mói với ảnh 4 và khẳng định rằng phương pháp mới có thể tăng độ chính xác của tất cả các lớp phủ, đặc biệt là nhận dạng các đối tượng có kích thước nhỏ hơn hoặc tương đương 1pixel trên ảnh phổ.

\section{Giới thiệu chung}

Đối với các phương pháp phân loại cứng hay các phương pháp phân loại thông thường dựa trên giả thiết rằng mỗi pixel chỉ có thể thuộc về một lớp phủ. Tuy nhiên, trên thực tế thì trong cùng một pixel có thể chứa nhiều lớp phủ khác nhau (Fisher và cộng sự., 1997)[7]. Trong mỗi tấm ảnh viễn thám, số lượng các pixel chứa nhiều lớp phủ khác nhau sẽ chiếm đa số, ví dụ: các pixel nằm trên đường biên của hai lớp đối tượng khác nhau hay các pixel chứa các đối tượng hình tuyến có kích thước nhỏ hơn 1 pixel, tạm gọi các pixel này là các pixel pha trộn (hình 1). Do vậy, việc phân loại dựa trên nguyên tắc mỗi pixel chỉ thuộc về một lớp phủ sẽ dẫn đến sai số nhất định và không thỏa mãn các yêu cầu thực tế về độ chính xác. (Xem hình 1)

Đối với các phương pháp phân loại mềm, mỗi pixel sẽ không chỉ thuộc về một lớp phủ mà có thể thuộc về nhiều lớp phủ và mỗi lớp phủ sẽ chiếm tỷ lệ phần trăm trong mỗi pixel. Phương pháp này được chứng minh là cho độ chính xác cao hơn các phương pháp phân loại cứng (Foodyvà cộng sự., 1996) [8]. Cách tiếp cận này cho phép mỗi pixel sẽ mang một giá trị thành phần giữa 0 và 1 cho mỗi lớp thay vì chỉ mang các giá trị 0 hoặc 1 như trong phân loại cứng. Như vậy đầu ra của phân loại mềm không phải là một bản đồ lớp phủ mà là một tập các lớp ảnh raster khác nhau, mỗi lớp đại diện cho một lớp phủ và giá trị của lớp thể hiện diện tích (hoặc phần trăm) của lớp phủ đó trong mồi pixel (Hình 2).
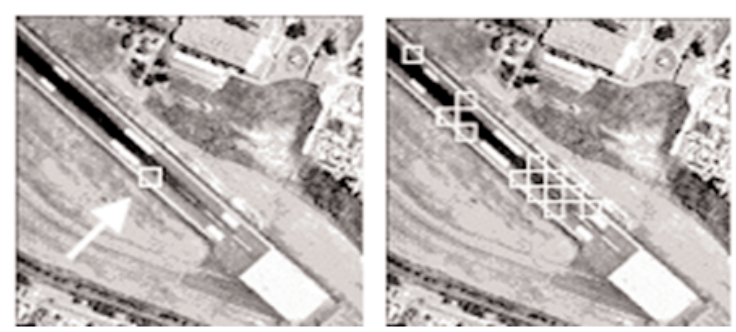

Hình 1: Mô tả hình ảnh các pixel pha trộn (nguồn tì [4])

Ngày nhận bài: 03/4/2019, ngày chuyển phản biện: 09/4/2019, ngày chấp nhận phản biện: 19/4/2019, ngày chấp nhận đăng: 29/4/2019 


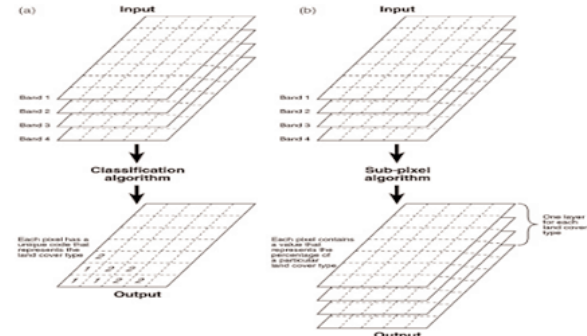

Hình 2: (a) Kêt quả của phân loại cúng,

(b) Kết quả của phân loại mềm (nguồn tù [5])

\section{Nội dung, phương pháp nghiên cứu}

\subsection{Phương pháp phân loại mềm sử dụng neuron Hopfield}

Mạng neuron Hopfield là mạng được kết nối hoàn toàn có hồi quy và chúng phần lớn được sử dụng cho việc liên kết tự động và tối ưu hoá. Những trạng thái cân bằng của mạng đạt được khi hàm năng lượng $(\mathrm{E})$ là cực tiểu. Những hàm năng lượng $E$ có thể được đơn giản hoá sao cho.

$$
E=-\frac{1}{2} \sum_{i} \sum_{j} w_{i j} v_{i} v_{j}-\sum_{i=1} v_{i} I_{i}
$$

Ở đây: $w_{i j}$ là trọng số kết nối từ neuron $\mathrm{j}$ tới $\mathrm{i}$

$v_{i}$ và $v_{j}$ là các đầu ra của neuron $\mathrm{i}$ và $\mathrm{j}$

$I_{i}$ là đường chéo bên ngoài hướng về phía neuron i.

Những thử nghiệm nghiên cứu của các tác giả trước đây cho thấy chỉ khoảng $60 \%$ kết quả đưa ra là gần đúng với vị trí thực tế của các tiểu pixel hình ảnh. (Xem hình 3)

Để khắc phục nhược điểm của một số phương pháp nêu trên, Nguyen Quang Minh, 2006[9] đã đưa ra phương pháp siêu phân giải bản đồ sử dụng mạng neuron (cụ thể là mạng neuron Hopfield) kết hợp ảnh toàn sắc tương ứng có độ phân giải không gian trung bình để tạo ra bản đồ lớp phủ mặt đất tiểu pixel ở mức siêu phân giải. Kết quả của phương pháp này cho thấy độ chính xác đạt được của bản đồ lớp phủ cao hơn so với các phương pháp siêu phân giải thông thường.

\subsection{Mô hình của phương pháp phân loại mềm sủ dụng neuron Hopfield}

Phương pháp này sử dụng ảnh toàn sắc như là thông tin bổ sung ở độ phân giải không gian trung bình để tạo ra một bản đồ độ phủ mặt đấtcó độ phân giải cao hơn độ phân giải gốc của ảnh. Để thực hiện điều này, có thể sử dụng ảnh toàn sắc cho bản đồ lớp phủ mặt đất, một mô hình thuận, một mô hình tổng hợp phổ và một mô hình đảo được đưa vào tạo thành một hàm điều kiện của ảnh toàn sắc trong mạng neuron Hopfield phát triển bởi Tatem và cộng sự., 2001a [13]. Hàm điều kiện của ảnh toàn sắc dựa trên nguyên tắc sự khác biệt về độ xám tạo ra bởi các tiểu điểm ảnh và độ chính xác trên ảnh toàn sắc là nhỏ nhất.

Phương pháp kết hợp ảnh toàn sắc vào quá trình lập bản đồ siêu phân giải lớp phủ sử dụng mạng neuron Hopfield được minh họa tại hình 4. Mô hình được thực hiện như sau: Từ các ảnh phổ gốc độ phân giải không gian (ví dụ: độ phân giải $20 \mathrm{~m}$ ), sử dụng phương pháp phân loại mềm ta thu được bản đồ lớp phủ với độ phân giải không gian là $20 \mathrm{~m}$. Từ kết quả phân loại mềm, sử dụng thuật toán siêu phân giải mạng neuron Hopfield có thể tạo ra bản đồ lớp phủ với độ phân giải không gian $5 \mathrm{~m}$. Từ bản đồ lớp phủ này sử dụng thuật toán trong mô hình thuận để tạo ra bản đồ lớp phủ ảnh đa phổ tổng hợp có độ phân giải không gian $5 \mathrm{~m}$, qua quá trình tổng hợp phổ ta sẽ nhận được bản đồ lớp phủ ảnh đa phổ tổng hợp có độ phân giải không gian $10 \mathrm{~m}$, tiếp tục quá trình tổng hợp phổ kết quả là tạo ra ảnh toàn sắc tổng hợp có độ phân giải không gian $10 \mathrm{~m}$. Sau đó sử dụng phép so sánh giữa ảnh toàn sắc gốc có độ phân giải không gian $10 \mathrm{~m}$ với ảnh toàn sắc tổng hợp được, khi đó kết quả sẽ đưa ra các sai số ảnh trên bản đồ lớp phủ ảnh toàn sắc thu nhận, sử dụng thuật toán mô hình đảo để giảm bớt các sai số ảnh.Tiếp tục sử dụng mạng neuron Hopfield cho quá trình tối ưu hóa đó đến khi nào sai số ảnh là tối thiểu thì quá trình sẽ dừng và kết quả đưa ra là bản đồ lớp phủ siêu phân giải tại độ phân giải không gian $5 \mathrm{~m}$ cho phép tạo ra ảnh toàn sắc tổng hợp gần giống như ảnh toàn sắc gốc thu được. (Xem hình 4)

2.3. Thục nghiệm sử dụng ảnh SPOT4 khu vục Lang Son 
Để kiểm nghiệm thuật toán trình bày trong phần trước, chúng tôi đã tiến hành thực sử dụng ảnh SPOT4. Để đánh giá thuật toán một cách chính xác, cần có các dữ liệu như sau: Bản đồ lớp phủ mặt đất với độ phân giải cao, ảnh phổ. Trong trường hợp sử dụng ảnh 4 , thì độ phân giải không gian của bản đồ lớp phủ dùng để đánh giá thuật toán phải cao hơn $20 \mathrm{~m}$ vì ảnh SPOT4 có độ phân giải là $20 \mathrm{~m}$ (đối với những pixel ở tâm của ảnh). Chúng tôi tiến hành thử thuật toán siêu phân giải sử dụng ảnh đa phổ giảm độ phân giải từ $20 \mathrm{~m}$ xuống còn $60 \mathrm{~m}$ để tạo ra bản đồ lớp phủ ở độ phân giải $60 \mathrm{~m}$, sau đó tiến hành phân loại mềm để tạo ra ảnh tỷ lệ thực và qua siêu phân giải bằng mạng neuron Hopfield tạo bản đồ ảnh tiểu pixel (20m). Bản đồ lớp phủ này được so sánh với dữ liệu kiểm tra đã có ở độ phân giải $20 \mathrm{~m}$ nhằm đánh giá khả năng ứng dụng của thuật toán. Để so sánh với các phương pháp khác, kết quả của thuật toán cũng được so sánh về độ chính xác đạt được với các phương pháp như phân loại cứng, đối với ảnh 20m, phân loại mềm.

Việc sử dụng ảnh giảm độ phân giải còn cho phép đánh giá ảnh hưởng của sai số định vị (georeferencing) đến kết quả của quá trình siêu phân giải sử dụng ảnh toàn sắc bằng mạng neuron Hopfield. Vì thuật toán mới sử dụng một loại ảnh là ảnh phổ nên việc sai số định vị ảnh có ảnh hưởng rất lớn tới kết quả. Với ảnh Spot4 giảm độ phân giải, thực nghiệm được thực hiện theo bốn bước như sau: (a) Dữ liệu kiểm tra và huấn luyện, (b) Giảm độ phân giải ảnh (c) Quá trình tiền xử lý (Phân loại mềm), và (d) Siêu phân giải bản đồ (hình 5).

\subsubsection{Dũ liệu kiểm tra. (Xem hình 6)}

Dữ liệu thô: Ảnh 4 với phân giải không gian 20m (đa phổ) được thu nhận trên một diện tích của khu vực tỉnh Lạng Sơn. Khu vực thực nghiệm được thực hiện diện tích nhỏ 480 x 480 pixel của ảnh của ảnh đa phổ (hình 6). Qua khảo

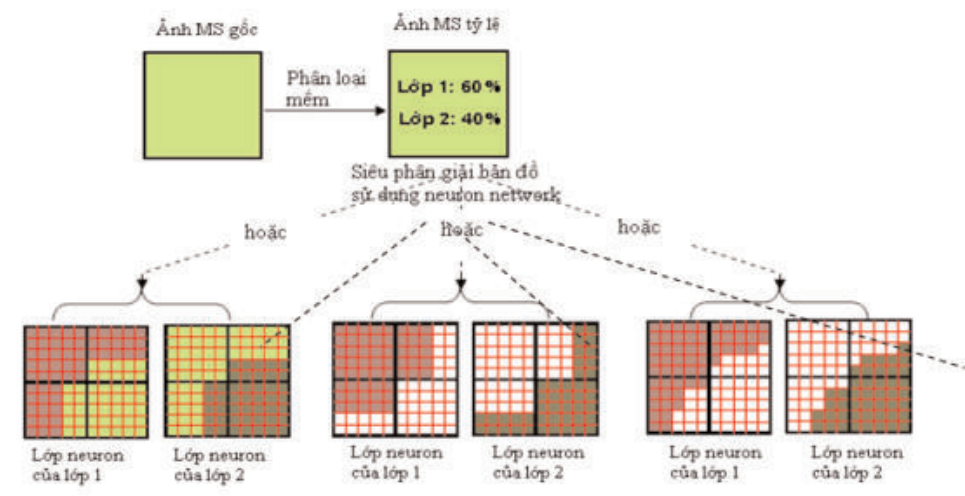

Hình 3: Một số kết quả của siêu phân giải

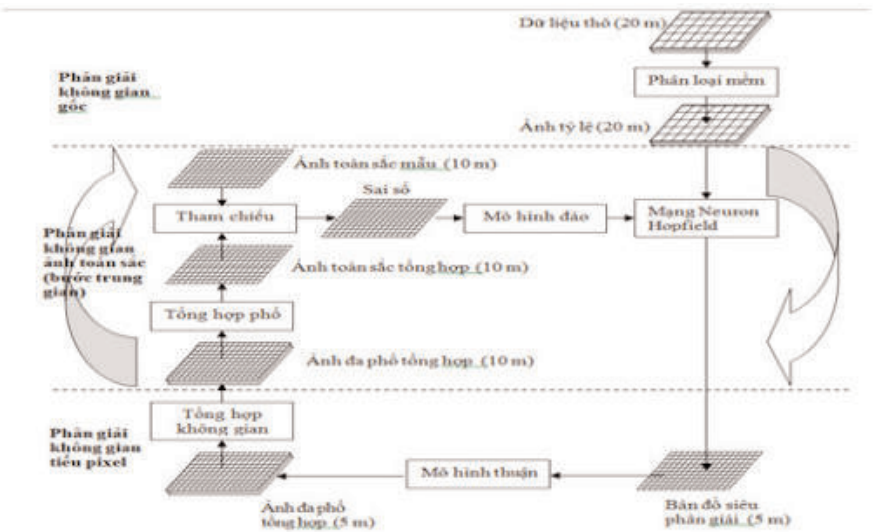

Hình 4: Siêu phân giải bản đồ mạng neuron Hopfield sử dụng ảnh toàn sắc (nguồn tì̀ [9]) 
sát, có năm lớp phủ trên khu vực này được nhận dạng là đường, đô thị, nước, nông nghiệp và đất rừng.

Dữ liệu: Để thực hiện quá trình phân loại mềm cần phải có dữ liệu mẫu. Trong nghiên cứu này, phân loại mềm đã thực hiện ở ảnh phổ với độ phân giải không gian là $60 \mathrm{~m}$ với dữ liệu mẫu được sử dụng là bản đồ lớp phủ ở độ phân giải $120 \mathrm{~m}$ được tạo ra bằng cách giảm độ phân giải ảnh lớp phủ 20m xuống 6 lần. (Xem hình 7)

\subsubsection{Giảm độ phân giải}

Ảnh đa phổ (20 m): Ảnh đa phổ 4 ở độ phân giải không gian $20 \mathrm{~m}$ đã được làm giảm độ phân giải xuống ba lần nhằm tạo ra một ảnh đa phổ ở độ phân giải không gian $60 \mathrm{~m}$. Ảnh đa phổ này sau đó được dùng để tạo ra các bản đồ lớp phủ ở độ phân giải không gian $60 \mathrm{~m}$ bằng phương pháp phân loại mềm (Hình 8).

\subsubsection{Phân loại mềm}

Một trường hợp phân loại $k$-nn dùng cho phân loại mềm với $k=5$, ảnh tỷ lệ lớp phủ được tạo ra với tỷ lệ sai số diện tích tổng thể là $P=$ $0.0101 \%$ và lỗi sai số trung phương tổng thể $1.4477 \%$ pixel. Kết quả phân loại mềm của 5 lớp phủ được thể hiện tại hình 9, 10, 11, bảng 1,2

\subsection{Kết quả, đánh giá}

Để đánh giá sự hiệu quả của thuật toán mới với 4 giảm độ phân giải, các kết quả đạt được của hai phương pháp: phân loại cứng với siêu phân giải sử dụng mạng neuron Hopfield thông thường so sánh một cách trực tiếp. Bản đồ lớp phủ được thành lập từ phương pháp phân loại cứng có độ phân giải $20 \mathrm{~m}$ được trình bày trên hình 10. Kết quả áp dụng siêu phân giải bản đồ sử dụng mạng neuron Hopfield theo phương pháp của Tatern và các cộng sự., (2001a) [13], cho các bản đồ lớp phủ với độ phân giải không gian trên hình 11. Bản đồ này là kết quả đạt được từ các kết quả phân loại mềm ở độ phân giải $60 \mathrm{~m}$ với tỷ lệ phóng là 3 lần, các hằng số trọng số $\mathrm{k}_{1}$ $=100, \mathrm{k}_{2}=100$ và $\mathrm{k}_{3}=150$, và 1000 vòng lặp.

Các phương pháp được đánh giá bằng cách so sánh dựa trên các ma trận sai số cũng như các tham số như Kappa Index Agreement (KIA), và sai số tổng hợp được trình bày trong các Bảng 1 và Bảng 2.

Ưu điểm của thuật toán mới được chứng minh bởi các số liệu thống kê về độ chính xác. Độ chính xác tổng thể của bản đồ lớp phủ tăng từ $62.99 \%$ với phân loại cứng lên $67.37 \%$ trong siêu phân giải bản đồ.

Độ chính xác của kết quả được đánh giá dựa trên ma trận sai số và các tham số thường được sử dụng để đánh giá đô chính xác như giá trị KIA, độ chính xác tổng thể, các sai số bỏ sót và sai số đọc nhầm.

Kết quả đánh giá định tính và định lượng đều khẳng định rằng phương pháp mới có thể tăng độ chính xác của tất cả các lớp phủ, đặc biệt là nhận dạng các đối tượng có kích thước nhỏ hơn hoặc tương đương 1 pixel trên ảnh phổ.

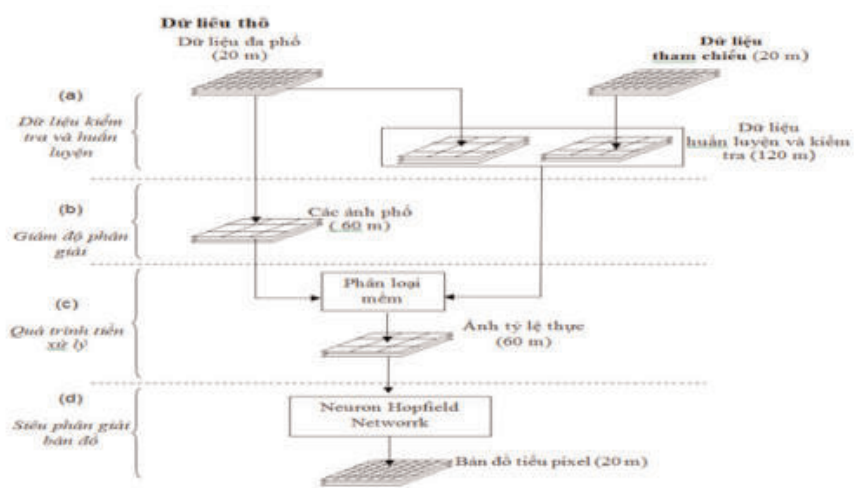

Hình 5: Bốn buớc trong thực nghiệm:(a) Dũ liệu kiểm tra và huấn luyện, (b) Giảm độ phân giải, (c) Quá trình tiền xủ lý, (d) Siêu phân giải bản đồ 

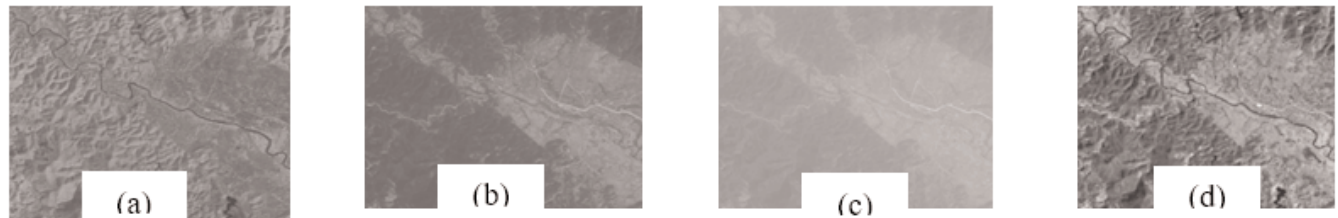

Hình 6: Các ảnh đa phổ với 4 kênh (a) Lục, (b) Đỏ, (c) Hồng ngoại, và (d) Hồng ngoại

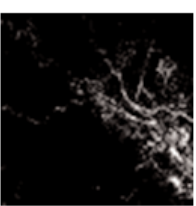

(a) Đường

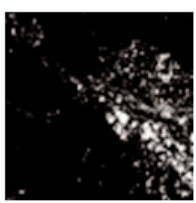

(b) Đô thị

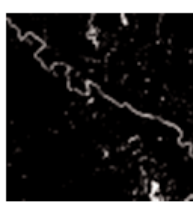

(c) Nước

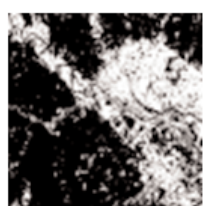

(d) Nông nghiệp

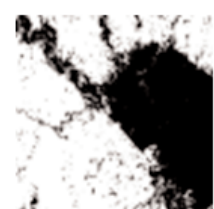

(e) Đất rừng

Hình 7: Năm lớp huấn luyện có độ phân giải 120 m

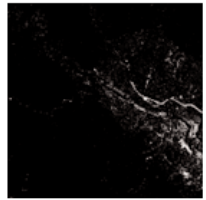

(a) Đường

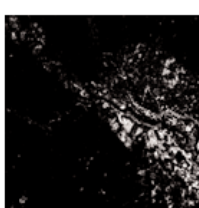

(b) Đô thị

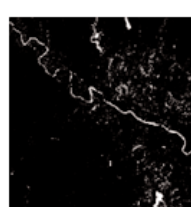

(c) Nước

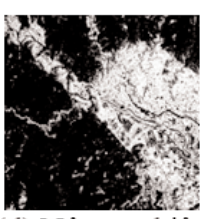

(d) Nông nghiệp

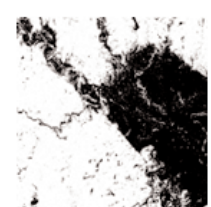

(e) Đất rừng

Hình 8: Năm lớp huấn luyện có độ phân giải $60 m$

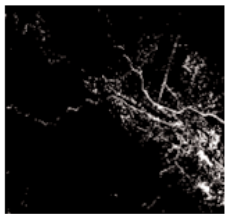

(a) Đường

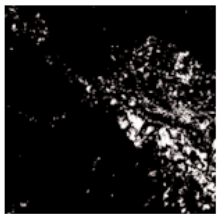

(b) Đô thị

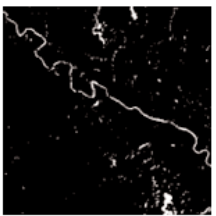

(c) Nước

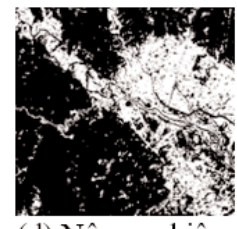

(d) Nông nghiệp

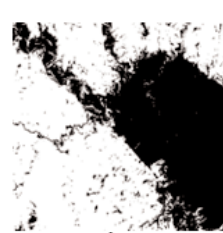

(e) Đất rừng

Hình 9: Kết quả phân loại mềm

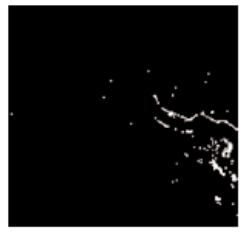

(a) Đường

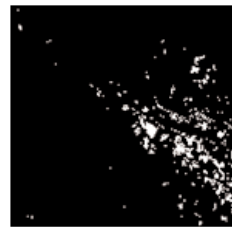

(b) Đô thị

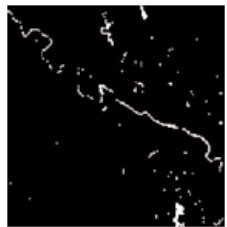

(c) Nước

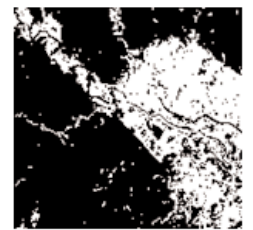

(d) Nông nghiệp

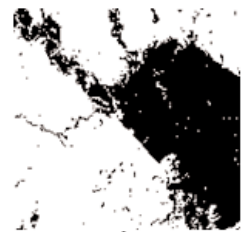

(e) Đất rừng

Hình 10: Kết quả của phân loại cúng bằng phưong pháp xác suất lớn nhất

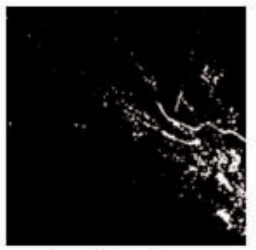

(a) Đường

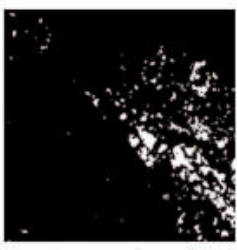

(b) Đô thị

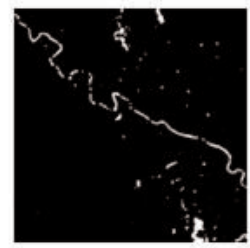

(c) Nước

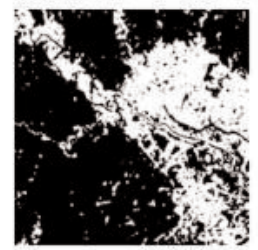

(d) Nông nghiệp

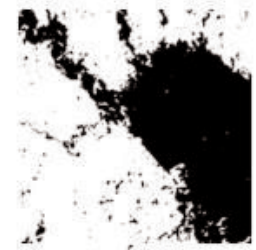

(e) Đất rừng

Hình 11: Kết quả của siêu phân giải sủ dụng mạng neuron Hopfield 
Bảng 1: Số liệu thống kê độ chính xác của kết quả phân loại ảnh đa phổ 4 giảm độ phân giải tính tù kết quả phân loại cứng

Ma trận sai số tính từ kết quả phân loại cứng

\begin{tabular}{|c|c|c|c|c|c|c|c|}
\hline $\begin{array}{r}\text { Ảnh tham } \\
\text { chiếu } \\
\text { Ảnh phân loại }\end{array}$ & Đường & Đô thị & Nước & $\begin{array}{l}\text { Nông } \\
\text { nghiệp }\end{array}$ & Đất rừng & $\begin{array}{l}\text { Tồng } \\
\text { pixel }\end{array}$ & $\begin{array}{c}\text { Sai số } \\
\text { đọc } \\
\text { nhầm }\end{array}$ \\
\hline Đường & 1743 & 1229 & 24 & 5544 & 17 & 8557 & 0.1773 \\
\hline Đô thi & 277 & 6356 & 7 & 6680 & 6 & 13326 & 0.1960 \\
\hline Nước & 0 & 1 & 2668 & 741 & 1557 & 4967 & 0.1242 \\
\hline Nông nghiệp & 147 & 649 & 1081 & 60320 & 10259 & 72456 & 0.3391 \\
\hline Đất rừng & 2 & 0 & 144 & 1388 & 129560 & 131094 & 0.2409 \\
\hline Tồng pixel & 2169 & 8235 & 3924 & 74673 & 141399 & \multirow{2}{*}{\multicolumn{2}{|c|}{$\begin{array}{l}\text { KIA trung bình } \\
62.99\end{array}$}} \\
\hline Sai sổ bỏ sót & 0.0314 & 0.0384 & 0.0154 & 0.1150 & 0.0580 & & \\
\hline
\end{tabular}

Bảng 2: Số liệu thống kê độ chính xác của kết quả phân loại ảnh đa phổ 4 giảm độ phân giải tính tù̀ kết quả siêu phân giải

Ma trận sai số tính từ kết quả siêu phân giải

\begin{tabular}{|c|c|c|c|c|c|c|c|}
$\begin{array}{r}\text { Ảnh tham } \\
\text { chiếu } \\
\text { Ảnh phân lợi }\end{array}$ & Đường & Đô thị & Nước & $\begin{array}{c}\text { Nông } \\
\text { nghiệp }\end{array}$ & Đất rừng & $\begin{array}{c}\text { Tồng } \\
\text { pixel }\end{array}$ & $\begin{array}{c}\text { Sai số } \\
\text { đọc } \\
\text { nhầm }\end{array}$ \\
\hline Đường & 4340 & 947 & 13 & 3218 & 39 & 8557 & 0.1585 \\
\hline Đô thị & 695 & 9742 & 8 & 2880 & 1 & 13326 & 0.1557 \\
\hline Nước & 12 & 1 & 3521 & 737 & 696 & 4967 & 0.0918 \\
\hline Nông nghiệp & 859 & 1053 & 294 & 66441 & 3809 & 72456 & 0.2600 \\
\hline Đất rừng & 5 & 1 & 179 & 2724 & 128185 & 131094 & 0.1799 \\
\hline Tồng pixel & 5911 & 11744 & 4015 & 76000 & 132730 & \multicolumn{2}{c|}{ KIA trung bình } \\
\hline Sai số bó sót & 0.0251 & 0.0242 & 0.0084 & 0.0676 & 0.0324 & \multicolumn{2}{|c|}{67.37} \\
\hline
\end{tabular}

\section{Kết luận}

Mạng neuron Hopfield thực sự là một công cụ hữu ích trong việc thực hiện bài toán tối ưu hoá trong việc xác định vị trí của các lớp phủ trong mỗi pixel theo kết quả của phân loại mềm và đã cho các kết quả tốt.

Mạng neuron Hopfield cho phép dễ dàng đưa vào những thông tin bổ sung để tăng độ chính xác và độ phân giải không gian của bản đồ lớp phủ. Bản đồ lớp phủ ở dạng raster đạt được thông qua siêu phân giải sử dụng mạng neuron Hopfield cho kết quả với độ chính xác cao hơn hẳn so với các phương pháp phân loại cứng.

Kết quả thực nghiệm cũng cho thấy sai số nắn chỉnh hình học (định vị) ảnh toàn sắc có ảnh hưởng đến kết quả siêu phân giải sử dụng mạng neuron Hopfield. Tuy nhiên, ở mức độ sai số trung phương nắn chỉnh hình học nhỏ hơn hoặc bằng 1 pixel, phương pháp mới vẫn cho kết quả cao hơn so với các phương pháp truyền thống.

Qua khảo sát thực tế, chúng tôi nhận thấy rằng các điểm ảnh này hầu hết là các điểm ảnh pha trộn. Nếu áp dụng thuật toán siêu phân giải có thể tăng độ phân giải không gian cũng như xác định đường biên của các địa vật một cách chính xác, do đó cho phép nắn chỉnh hình học với độ chính xác cao hơn. $\bigcirc$

\section{Tài liệu tham khảo}

[1]. Đặng Thanh Tùng (2012), Phương pháp phân loại lớp phủ bề mặt sừ dụng ảnh cường độ phản hồi tì dũ liệu Lidar kết hợp với ảnh viễn thám, Tạp chí Khoa học đo đạc và Bản đồ số 12 tháng 6/2012 - trang 29 -36.

[2]. Lương Chính Kế, Chiến lược phát triển ảnh vệ tinh có độ phân giải siêu cao của $M \tilde{y}$, trường ĐH Bách khoa Vacsava.

[3]. Andrew J Tatem, Hugh G Lewis, Peter M Atkinson, Mark S Nixon., 2001, Multiple-class land-cover mapping at the sub-pixel scale using a Hopfield neural networkInternational Journal of Applied Earth Observation and Geoinformation, Volume 3, Issue 2, pp 184-190

[4]. Anna Haglund (2000),Towards soft classification of satellite data, Sweden.

[5]. Atkinson, P. M., Cutler, M. E. J., and 
Lewis, H (1997), Mapping sub-pixel proportional land cover with AVHRR imagery, International Journal of Remote Sensing 18, pp. 917-935.

[6]. Foody, G. M., and Cox, D. P (1994), Subpixel land cover composition estimation using a linear mixture model and fuzzy membership functions, International Journal of Remote Sensing 15, pp. 619-631.

[7]. Fisher, P., 1997. The pixel: a snare and a delusion. International Journal of Remote Sensing 18: 679-685.

[8]. Giles M. Foody, Manoj K. Arora., 1996, Incorporating mixed pixels in the training, allocation and testing stages of supervised classifications, Pattern Recognition Letters, pp 13891398.

[9]. Nguyen Quang Minh (2006), Super-resolution mapping using hopfield neural network with supplementary data, University of Southampton.

[10]. Marzieh Mokarrama, Majid, Volume 21, April 2018, HojatiLandform classification using a sub-pixel spatial attraction model to increase spatial resolution of digital elevation model (DEM), The Egyptian Journal of Remote Sensing and Space Science, pp 111-120.
[11]. Rahel Hailu Kassaye (2006), Suitability of Markov Random Field-based Method for Super-Resolution Land Cover Mapping, The Netherlands.

[12]. Shangrong Wu,Jianqiang Ren, Zhongxin Chen, Wujun Jin,Volume 210, 2018 Influence of reconstruction scale, spatial resolution and pixel spatial relationships on the sub-pixel mapping accuracy of a double-calculated spatial attraction model, Remote Sensing of Environment, pp 345-361.

[13]. Tatem, A . J., Lewis, H .G., Atkinson, P. M., and Nixon, M . S., 2001b,Super-Resolution Target Identification from Remotely Sensed Images using a Hopfield Neural Network, IEEE Transactions on Geoscience and Remote Sensing, pp. 781-796.

[14]. Tatem, A . J., Lewis, H .G., Atkinson, P. M., and Nixon, M . S (2003),Increasing the spatial resolution of agricultural land cover maps using a Hopfield neural network, Int. J. Geographical information science.

[15]. Verhoye, J., and De Wulf, R (2002), Land cover mapping at sub-pixel scales using linear optimization techniques, Remote Sensing of Environment. $\mathrm{O}$

\section{Summary}

Using the Hopfield neuron network enhances the accuracy of identifying objects smaller than 1 pixel in the spectral image

Dang Thanh Tung, State Key Laboratory of Information Engineering in Surveying, Mapping and Remote Sensing (LIESMARS), Wuhan University, 129 Luoyu Road, Wuhan 430079, China/ Hanoi University of Natural Resources and Environment

Do Nhu Hiep, Pham Dinh Tinh, Hanoi University of Natural Resources and Environment

Do Manh Ha, Vietnam Department of Surveying and Geographic Information

Nguyen The Luan, Institute of Marine Geology and Geophysics - Vietnam Academy of Science and Technology

In the field of remote sensing, soft classification is considered to have higher accuracy than hard classification. For the super-resolution, the Hopfield neuron network was used to perform an optimization problem in determining the position of the layer in each pixel. This study evaluates the effectiveness of the new algorithm with the 4 image and the new method can increase the accuracy of all layers, especially those of smaller 1 pixel. $\bigcirc$ 